\title{
Two-step identification of taro (Colocasia esculenta cv. Xinmaoyu) using specific psbE- pet $L$ and simple sequence repeat-sequence characterized amplified regions (SSR-SCAR) markers
}

\author{
H.J. Dai ${ }^{1 *}$, Y.M. Zhang ${ }^{2 *}$, X.Q. Sun ${ }^{2}$, J.Y. Xue ${ }^{2}$, M.M. Li ${ }^{2}$, M.X. Cao ${ }^{1}$, \\ X.L. Shen ${ }^{1}$ and Y.Y. Hang ${ }^{2}$ \\ ${ }^{1}$ Seed Administrative Station of Suzhou, Suzhou, China \\ ${ }^{2}$ Institute of Botany, Jiangsu Province and Chinese Academy of Sciences, \\ Nanjing, China \\ *These authors contributed equally to this study. \\ Corresponding authors: X.L. Shen / Y.Y. Hang \\ E-mail: szseed@126.com / hangyueyu@cnbg.net
}

Genet. Mol. Res. 15 (3): gmr.15038108

Received November 19, 2015

Accepted March 15, 2016

Published August 5, 2016

DOI http://dx.doi.org/10.4238/gmr.15038108

Copyright (C) 2016 The Authors. This is an open-access article distributed under the terms of the Creative Commons Attribution ShareAlike (CC BY-SA) 4.0 License

\begin{abstract}
Colocasia esculenta cv. Xinmaoyu is an eddoe-type taro cultivar local to Taicang, Jiangsu Province, China; it is characterized by its pure flavor, glutinous texture, and high nutritional value. Due to its excellent qualities, the Trademark Office of the State Administration for Industry and Commerce of the People's Republic of China awarded Xinmaoyu, a geographical indication certification in 2014. Therefore, there is an urgent need to develop an efficient molecular marker for the specific identification of this cultivar, which would greatly facilitate the
\end{abstract}


conservation and utilization of this unique germplasm resource. In the present study, amplifying the psbE-petL fragment from two dasheentype and seven eddoe-type taro cultivars revealed three conserved insertions/deletions among sequences from the two taro types. Based on these sequence differences, a pair of site-specific primers was designed targeting the psbE-petL sequence from the dasheen-type taro, which specifically amplified a DNA band in all individuals from cultivars of this type, but not in those from the seven eddoe-type cultivars. To discriminate Xinmaoyu from the other eddoe-type taro cultivars, a pair of simple sequence repeat-sequence characterized amplified region (SSR-SCAR) primers was further developed to specifically amplify a DNA band from all Xinmaoyu individuals, but not from individuals of other eddoe-type taro cultivars. In conclusion, through a twostep-screening procedure using psbE-petL and SSR-SCAR markers, we developed a pair of primers that could specifically discriminate Xinmaoyu from nine taro cultivars commonly cultivated in Jiangsu Province and Fujian Province.

Key words: Taro; Xinmaoyu; psbE-petL; SSR; SCAR; Two-step marker

\section{INTRODUCTION}

Taro [Colocasia esculenta (Linnaeus) Schott] is a perennial herb in the genus Colocasia of the family Araceae. This species is generally believed to originate from tropical regions in China, India, and the Malay Peninsula (Wu and Raven, 2010). It is reported that taro ranks 14th among staple crops (Nath et al., 2013). According to a survey by the Food and Agriculture Organization of the United Nations (FAO), the annual worldwide production of taro reached 10,016,012 tons, with a planting area of 1,432,333 hectares in 2014 (http://faostat3.fao.org). Although only one species of taro [C. esculenta (L). Schott] is documented in the Flora of China (Wu and Raven, 2010), taro possesses rich germplasm resources due to its long history of cultivation. The tillering habits of taro, which confer fitness to various habitats (Zhang and Yang, 1984), serve as a consistent characteristic for species discrimination. Thus, according to the tillering habits of the corm, taro is commonly classified into the three types: polycephalous dasheen, dasheen, and eddoe type. For the polycephalous dasheen type, the mother corm often forms tillers in a cluster. All corms connect to form a whole organ, and no major differences between the mother corm and daughter corms are observed. The dasheen type contains a single or several well-developed mother corms, with fewer and smaller daughter corms. The eddoe type contains a poorly developed mother corm and clustered daughter corms (Editorial Board of the Flora of China, 1979; Wu and Raven, 2010; Yang and Kong, 1998).

Previously, the identification of taro cultivars has been based on morphological characteristics (Zhang and Zhang, 1998; Li et al., 2004; Quero-Garcia et al., 2004; Huang et al., 2013, 2014). For example, taro from New Delhi could be identified in 260 taro cultivars by the leaf lamina orientation, with the tip pointing downwards and Jinggangshanyu could be identified by a continuous dark-brown strip on the leaf margin (Huang et al., 2013). However, morphological identification is highly subjective. Certain morphological features

Genetics and Molecular Research 15 (3): gmr.15038108 
are susceptible to the effects of environmental factors and significant variations have been observed. For example, plant height, and tuber size of one taro cultivar were found to vary at different altitudes (Trimanto et al., 2010). Hence, it would be unreliable to identify a specific cultivar based solely on morphological characteristics.

To overcome the disadvantages mentioned above, molecular markers have been widely applied to the investigation of plant diversity (Lu et al., 2011; Nunes et al., 2012; Lin et al., 2015). In recent years, researchers have attempted to differentiate or identify taro using molecular markers. Irwin et al. (1998) reported the molecular identification of 44 taro cultivars from several regions of Oceania using random amplified polymorphic DNA (RAPD) markers, which are able to differentiate various taro cultivars and taro from different geographical regions. For example, the cultivar Java48 produced a specific band that could be used to differentiate it from other cultivars. Primer D15 amplified a specific band from three cultivars from Papua New Guinea that could be used to differentiate them from cultivars from other regions. Nunes et al. (2012) analyzed seven taro cultivars in Brazil using simple sequence repeat (SSR) markers and found that these could be differentiated according to differences in the DNA band pattern obtained with the Xuqtem110 primer. Additionally, specific molecular markers based on amplified restriction fragment length polymorphism have been adopted to specifically identify two cultivars of taro, Ziganyu (purple stem taro) and Lvganyu (green stem taro) (Li et al., 2011). Since these molecular markers do not changed during different stages of plant growth and are not affected by environmental factors, they are more efficient at identifying specific cultivars compared to morphological markers.

C. esculenta cultivar Xinmaoyu is an excellent taro cultivar local to Taicang, Jiangsu Province, China. This cultivar belongs to the eddoe-type taro and is featured with pure flavor, glutinous texture, and high nutritional value (Chen et al., 2004). Due to these unique qualities, Xinmaoyu was awarded a geographical indication certification by the Trademark Office of the State Administration for Industry and Commerce of the People's Republic of China in 2014. At present, the identification of Xinmaoyu still relies heavily on traditionally morphological features (Chen et al., 2004; Jiang 2006). However, most of the edible parts of taro are their corms, which display high morphological similarities to other eddoe-type taro cultivars. Therefore, there is a need to develop a unique and specific molecular marker for Xinmaoyu authentication, which would facilitate the conservation of this excellent germ resource.

\section{MATERIAL AND METHODS}

\section{Extraction of total genomic DNA from plants}

Total genomic DNA was extracted from C. esculenta cultivar Xinmaoyu from Taicang, Jiangsu Province and eight additional taro cultivars commonly cultivated in Jiangsu Province and Fujian Province in China (Table 1). Briefly, $100 \mathrm{mg}$ fresh leaf tissue was collected from seedlings and disrupted using a Tissue Lyser LT (Qiagen, Germany). Total genomic DNA was extracted using the Easy Pure Plant Genomic DNA Kit (TransGen Biotech, Beijing, China). The concentration and purity of the extracted DNA were determined using a protein nucleic acid analyzer (Eppendorf, USA). The concentration of each DNA sample was adjusted to 20 $\mathrm{ng} / \mu \mathrm{L}$, and the samples were stored at $-20^{\circ} \mathrm{C}$ until analysis.

Genetics and Molecular Research 15 (3): gmr.15038108 
Table 1. Accessions sampled in the present investigation.

\begin{tabular}{l|l|l|c|l|l}
\hline Code & Accession & Properties & Number of plants & Type & Collection areas \\
\hline 1 & Xinmaoyu & Local variety & 8 & Eddoe type & Taicang, Jiangsu \\
\hline 2 & Suyouyu 1 & Bred variety & 8 & Eddoe type & Jingjiang, Jiangsu \\
\hline 3 & Xiangheyu & Local variety & 7 & Eddoe type & Taixing, Jiangsu \\
\hline 4 & Xiangshayu & Local variety & 6 & Eddoe type & Haimen, Jiangsu \\
\hline 5 & Yangyu 1 & Bred variety & 10 & Eddoe type & Yangzhou, Jiangsu \\
\hline 6 & Hongyayu & Local variety & 10 & Eddoe type & Yongtai, Fujian \\
\hline 7 & Hongxiangyu & Local variety & 7 & Eddoe type & Jintan, Jiangsu \\
\hline 8 & Xiangjiaoyu & Local variety & 4 & Dasheen type & Changshu, Jiangsu \\
\hline 9 & Longxiangyu & Local variety & 10 & Dasheen type & Xinghua, Jiangsu \\
\hline
\end{tabular}

\section{Amplification of a chloroplast DNA fragment}

The primers used to amplify the psbE-petL fragment from chloroplast DNA (F: AACGCGGTTTTGATCAGTAT, R: CAATTCATTGCGCAACTTGT) were previously used in two taro cultivars, C. esculenta var. GP and C. esculenta var. RR (Ahmed et al., 2013). The primers were synthesized by Nanjing Realgene Bio-Technologies, Inc. The PCR mixtures contained the following components: $20 \mathrm{ng}$ DNA template, reaction mix (containing $200 \mathrm{pmol}$ Tris- $\mathrm{HCl}, 1 \mu \mathrm{mol} \mathrm{KCl}, 30$ pmol MgCl, $4 \mu \mathrm{mol}$ dNTPs, and bromophenol blue), 10 pmol each of the forward and reverse primers, $1 \mathrm{U}$ Taq DNA polymerase, and double-distilled water to a total volume of $20 \mu \mathrm{L}$. The PCR conditions were as follows: pre-denaturation at $94^{\circ} \mathrm{C}$ for $7 \mathrm{~min}$, followed by 30 cycles of denaturation at $94^{\circ} \mathrm{C}$ for $1 \mathrm{~min}$, annealing at $50^{\circ} \mathrm{C}$ for $30 \mathrm{~s}$, and extension at $72^{\circ} \mathrm{C}$ for $45 \mathrm{~s}$, and a final extension step at $72^{\circ} \mathrm{C}$ for $10 \mathrm{~min}$. The PCR products were confirmed by $1.5 \%$ agarose gel electrophoresis containing ethidium bromide using $1 \mathrm{X}$ TAE buffer. Electrophoresis was performed at $80 \mathrm{~V}$ with the DNA marker DS2000 (Dongsheng Biotech, Guangzhou, China). After electrophoresis for $1 \mathrm{~h}$, the PCR products were examined and imaged using a gel documentation system (Tanon Science \& Technology Co., Ltd, Shanghai, China).

\section{Cloning and sequencing of PCR products}

The DNA band of interest was recovered and purified from the PCR products using an agarose gel DNA extraction kit (Generay Biotech, Shanghai, China). The recovered DNA fragments were ligated into the pMD19-T vector (TaKaRa Biotechnology, Co., Ltd., Dalian, China) according to the manufacturer instructions. The ligation products were transformed into Escherichia coli DH5a competent cells (TaKaRa Biotechnology, Co., Ltd., Dalian, China). Single colonies were selected and colony-PCR was performed with the universal primers M13 and RV-M. Clones producing the correct DNA band were sent to Nanjing Realgene BioTechnologies, Inc. for sequencing.

\section{Design and validation of chloroplast fragment-specific primers}

The sequencing results for the amplified chloroplast fragment were aligned using the ClustalW program in Mega 6.0 with default parameters (Tamura et al., 2013). Based on sequence differences among taro cultivars, a pair of site-specific primers F1 (5'-CCTGTGTTTGGTAATATTTCCT-3') and R1 (5'-TGTCATTGTATTCGGGTTCG-3') was 
designed using Primer 5.0 (Premier Biosoft Interpairs, Palo Alto, CA, USA). PCR validation was then performed for all individuals of the nine cultivars using the same reactions as described above. The PCR conditions were as follows: predenaturation at $94^{\circ} \mathrm{C}$ for $5 \mathrm{~min}$, followed by 30 cycles of denaturation at $94^{\circ} \mathrm{C}$ for $30 \mathrm{~s}$, annealing at $60^{\circ} \mathrm{C}$ for $30 \mathrm{~s}$, extension at $72^{\circ} \mathrm{C}$ for $45 \mathrm{~s}$, and a final extension step at $72^{\circ} \mathrm{C}$ for $8 \mathrm{~min}$.

\section{Screening of SSR primers}

SSR primers were selected from the literature (Lu et al., 2011; Sardos et al., 2012) and synthesized by Nanjing Realgene Bio-Technologies, Inc. PCR was performed using DNA pooled from all individuals from seven cultivars of the eddoe-type taro as a template. The reactions were prepared as mentioned above. The PCR conditions were as follows: predenaturation at $94^{\circ} \mathrm{C}$ for $5 \mathrm{~min}$, followed by 40 cycles of denaturation at $94^{\circ} \mathrm{C}$ for $45 \mathrm{~s}$, annealing at $50^{\circ} \mathrm{C}$ for $1 \mathrm{~min}$, extension at $72^{\circ} \mathrm{C}$ for $1 \mathrm{~min}$, and a final extension step at $72^{\circ} \mathrm{C}$ for $10 \mathrm{~min}$. The PCR products were checked by $2 \%$ agarose gel electrophoresis and stained with ethidium bromide. Electrophoresis was performed at $80 \mathrm{~V}$ for $1.5 \mathrm{~h}$, with the DNA marker DS2000.

\section{Primer design and verification based on SSR-sequence characterized amplified regions (SSR-SCAR) markers}

Primers that amplified a specific DNA band from Xinmaoyu were selected. Specific DNA bands were excised and recovered from an agarose gel. The recovered DNA fragments were cloned and sequenced. Using these sequencing results, a pair of specific primers $\mathrm{F} 2$ (5'-TTGTTCTCCCTTACCTCGTCG-3') and R2 (5'-GTGTGAGCTGATTGGGCCACA-3') was designed using Primer 5.0. The primers were used for PCR validation in all individuals from seven cultivars of the eddoe-type taro. The PCR mixtures were prepared as mentioned above. The PCR conditions were as follows: predenaturation at $94^{\circ} \mathrm{C}$ for $5 \mathrm{~min}$, followed by 30 cycles of denaturation at $94^{\circ} \mathrm{C}$ for $45 \mathrm{~s}$, annealing at $50^{\circ} \mathrm{C}$ for $45 \mathrm{~s}$, extension at $72^{\circ} \mathrm{C}$ for 1 min, and a final extension step at $72^{\circ} \mathrm{C}$ for $8 \mathrm{~min}$.

\section{RESULTS}

The alignment of psbE-petL fragment sequences obtained from all taro cultivars showed that there were significant differences in the fragments amplified from eddoe-type and dasheen-type taro. Compared to the fragment amplified from the dasheen type, the fragment amplified from the eddoe type contained 19- and 4-bp deletions at 109-127 and 766-769 bp, respectively, and a 10-bp insertion at 726-735 bp (Figure 1). The site-specific primers were validated in all plants from two cultivars of the dasheen type and seven cultivars of the eddoe type. A clear 390-bp DNA band was obtained from all plants of the dasheen type, while no such band was obtained from any eddoe-type plants (Figure 2). These results showed that the psbE-petL-specific primers were able to differentiate these two types of taro.

Sixteen pairs of SSR primers were used for PCR amplification of DNA pooled from seven cultivars of the eddoe type. Based on these results, the Xinmaoyu-specific primers, Taro02-F and Taro02-R, were selected from the screen. This primer pair was able to amplify

Genetics and Molecular Research 15 (3): gmr.15038108 


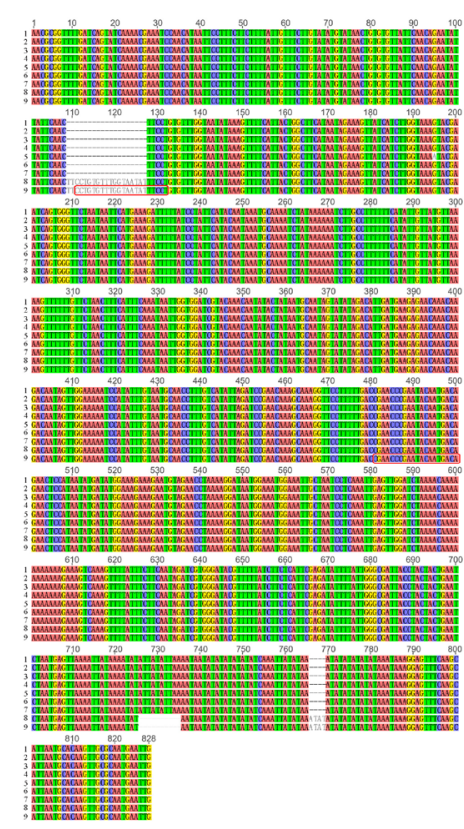

Figure 1. Alignment of sequences amplified from nine taro cultivars using psbE-petL primers. Sequences of the designed allele-specific primers used to distinguish between dasheen-type and eddoe-type taro are labeled by red boxes. Numbers 1-9 (in order) are Xinmaoyu, Suyouyu 1, Xiangheyu, Xiangshayu, Yangyu 1, Hongyayu, Hongxiangyu, Xiangjiaoyu, and Longxiangyu.
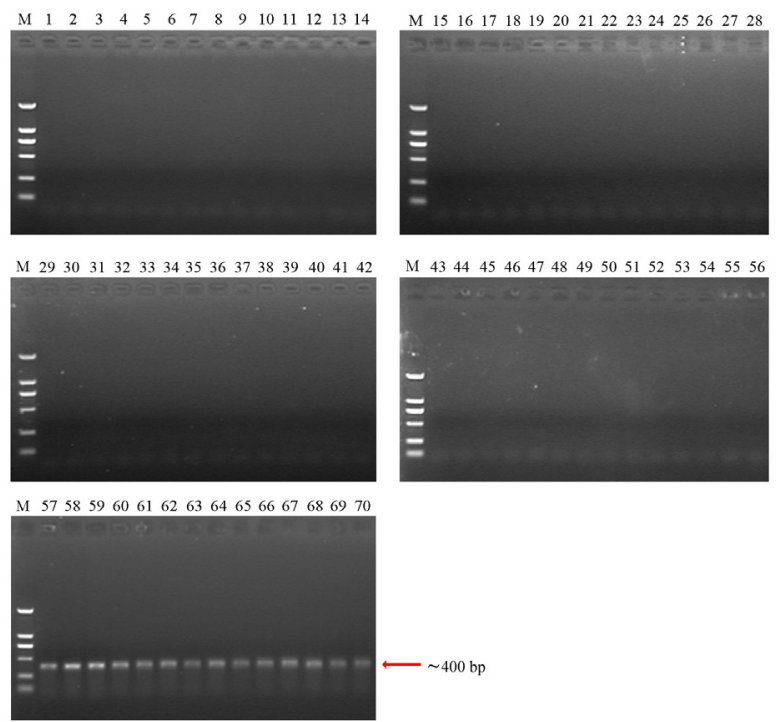

Figure 2. PCR verification of the allele-specific primers in all plants. Lanes 1-8 show PCR results from Suyouyu 1, 9-15 are from Xiangheyu, 16-21 are from Xiangshayu, 22-31 are from Yangyu 1, 32-41 are from Hongyayu, 4248 are from Hongxiangyu, 49-56 are from Xinmaoyu, 57-60 are from Xiangjiaoyu, 61-70 are from Longxiangyu. Lane $M=$ DS2000 DNA marker.

Genetics and Molecular Research 15 (3): gmr.15038108 
two specific DNA bands only from Xinmaoyu, which were approximately 750 and $400 \mathrm{bp}$ in size, respectively (Figure 3). The approximately 750-bp DNA fragment amplified from Xinmaoyu by the Taro02 primer pair was cloned and sequenced. The full-length DNA fragment contained 726 bp (the full sequence is shown in Figure 4). A pair of SCAR primers was designed to target this Xinmaoyu-specific fragment, and was subsequently validated in all plants from seven eddoe-type taro cultivars. A clear 478-bp DNA band of interest was amplified from all eight Xinmaoyu individuals. This specific band was not amplified from any individuals of the remaining six cultivars of the same type (Figure 5).

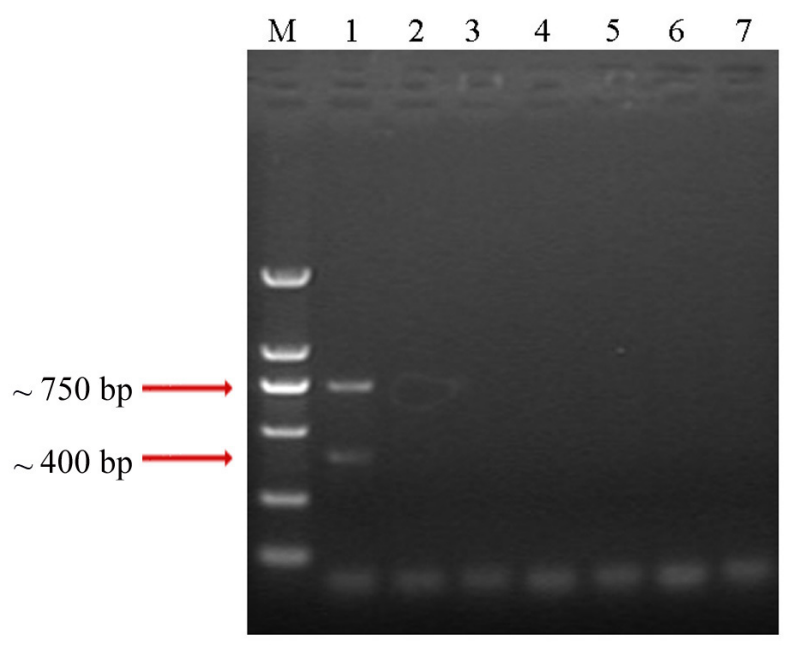

Figure 3. Agarose gel electrophoresis of PCR products obtained using the primer Taro02 in seven cultivars of eddoe-type taro. Lanes 1-7 (in order) are Xinmaoyu, Suyouyu 1, Xiangheyu, Xiangshayu, Yangyu 1, Hongyayu, and Hongxiangyu. Lane $M=$ DS2000 DNA marker.

ACTAATTCCGCAATACTTCACTATTTGATCATTGTTGTCATAGATTCATCTTTATATGTATT TTTTCGATCCAACATCCTAGAAGAAAACTAACATGAGGAACAAAGAACAGAGGAAAA AAAACTTACATGCCTTTTCGCCATAACAAATAGTTGGTTTTGACGGGTCTAGTCGCATTA TCTCTGGCGGGTCGTAAAGTTCCATCTTCGACGGGTCTGACTACGCCATTTCTGACGGG CATTGTTCTCCCTTACCTCGTCGATGAGACCTCCTCTGGCAAAAAATAAACTTTTCCAT TCTTTCATGTGGAGGACGATTTGGGGTCCATATGGACAACACGTTCTTTGGATGCCTTT GTCGTCCCTGGAAACCTTGAAGCAACTCTAGCGGGGGGGGGGGGGAGAGAGAGAGA GAGAGAGAGAGAGAGAGAGAGAGAGAGAGAGGAAGAATTGACTCGCCACCATGAAT CAATGGTAATGGCGTGAAGGGTATTTTTGGAAACAAACCCTATTGCTTGCTTAAATGCG ATGGGATGGAGTTCATCATTTTGAATCACGTATCCGGGAGGGGGGTTACGAAACGGGC GTAGGGGGTTATGGCACCATTCTCCATGCCGTTTGAAAATGGAGCGGCTAGTCATGAAA GGACACACCATTTGAGAGCCTCTTGGGTGCGTTGAGCTGTCAGACACGCTCCATGTGG CCCAATCAGCTCACACCCGGCGAT

Figure 4. Nucleotide sequence of the Xinmaoyu-specific fragment amplified using primer Taro02. Underlined sequences were used to design sequence characterized amplified regions (SCAR) primers. 

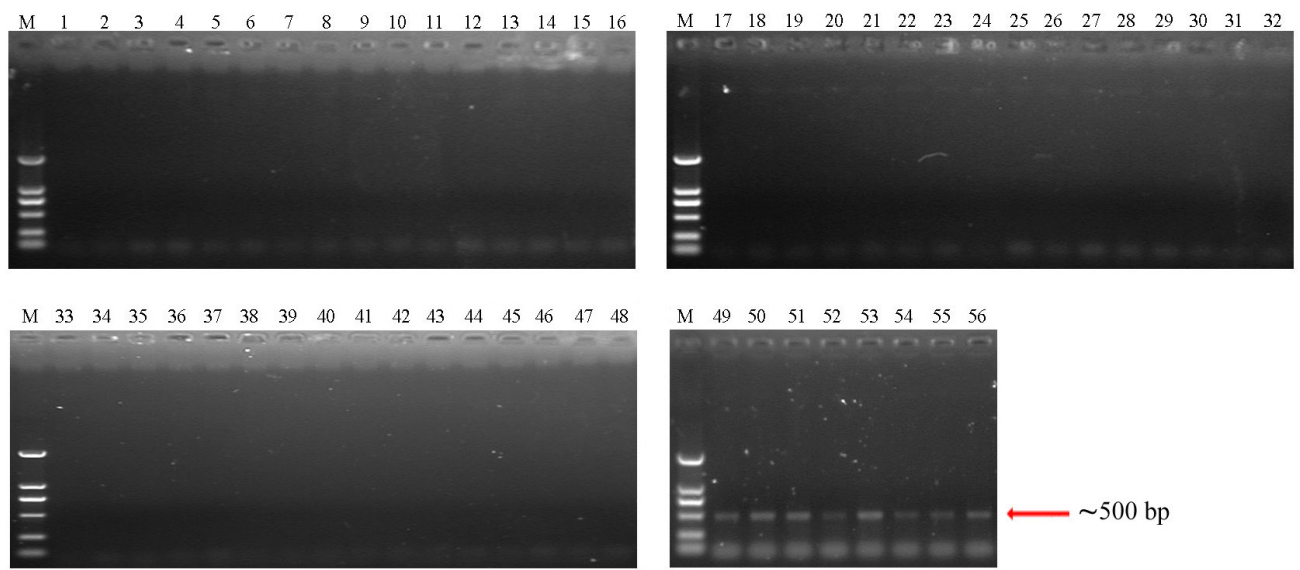

Figure 5. PCR verification of the simple sequence repeat-sequence characterized amplified region (SSR-SCAR) primers in all plants from seven cultivars of eddoe-type taro. Lanes 1-8 represent PCR results from Suyouyu 1, 9-15 are from Xiangheyu, 16-21 are from Xiangshayu, 22-31 are from Yangyu 1, 32-41 are from Hongyayu, $42-48$ are from Hongxiangyu, 49-56 are from Xinmaoyu. Lane $M=$ DS2000 DNA marker.

\section{DISCUSSION}

\section{Significance of the two-step development of Xinmaoyu-specific identification markers}

In this study, psbE-petL-based primers could amplify a specific DNA band from two cultivars of the dasheen-type taro. However, no such band was obtained from six cultivars of the eddoe-type taro. These findings suggest that this marker could be used to differentiate the two types of taro. Marked differences in morphology and chromosome ploidy, as well as differences at the molecular level, were observed between dasheen and eddoe types (Zhang and Yang, 1984; Huang et al., 2012). Studies at the molecular level also indicated that there are relatively independent genetic relationships between the two types of taro (Irwin et al., 1998). Phylogenetic analyses based on RAPD markers in 44 taro cultivars showed that dasheen and eddoe types are relatively distant and could form separate branches (Irwin et al., 1998; NusaifaBeevi et al., 2011). The present study showed that the psbE-petL marker may be capable of differentiating dasheen and eddoe types, within which genetic divergence has occurred.

Since the marker described above could not identify Xinmaoyu, the second step of the present study was to screen Xinmaoyu from the other six cultivars of the eddoe-type taro using SSR markers. Specific primers were obtained that could only amplify the DNA band of interest from Xinmaoyu, which were subsequently converted to SCAR markers. Validation of these markers revealed that no DNA band was amplified from the other six cultivars of the eddoe-type taro. This result indicated that the SSR-SCAR markers developed in the present study could specifically differentiate Xinmaoyu from other cultivars of the eddoe type.

This two-step identification process is not without precedent. Indeed, 16S rDNA markers were first used to identify Yersinia enterocolitica and Y. pseudotuberculosis; and 
then a PCR-RFLP technique was applied to specifically identify strain 1B/O8 from all other strains of $Y$. enterocolitica (Wołkowicz et al., 2014). The success of Wołkowicz and colleagues demonstrated that it is feasible to establish two-step molecular markers for use in identification. Therefore, the results of the present study also support the proposed twostep identification method based on psbE-petL and SSR-SCAR markers for the specific identification of Xinmaoyu.

\section{Efficiency evaluation of Xinmaoyu-specific molecular identification markers}

Xinmaiyu, a taro landrace protected under the Geographical Indication of China, has excellent qualities and is in great demand in the Chinese market. However, it is difficult to distinguish this cultivar from other eddoe-type taro cultivars based on morphological characteristics, especially for the edible corms after separation from the whole plant. In this study, we developed site-specific PCR markers for the identification of Xinmaoyu, based on psbE-petL and SSR-SCAR markers. The specific markers differentiated Xinmaoyu from eight additional cultivars of taro commonly cultivated in Jiangsu and Fujian provinces; the identification efficiency was $100 \%$. Compared to traditional methods based on morphology, physicochemical indicators, or non-specific molecular markers, the proposed PCR-based identification technique is accurate, reproducible, stable, and reliable. The development of specific molecular markers for Xinmaoyu, an excellent cultivar local to Taicang, provides guidance for germplasm identification and the innovation of other valuable taro landraces.

\section{Conflicts of interest}

The authors declare no conflict of interest.

\section{ACKNOWLEDGMENTS}

Research supported by the Foundation Project of Suzhou Municipal Science and Technology Bureau (\#SZP201313) and the Ability Improvement Project of Jiangsu Social Scientific Research Institutions (\#BM2015019).

\section{REFERENCES}

Ahmed I, Matthews PJ, Biggs PJ, Naeem M, et al. (2013). Identification of chloroplast genome loci suitable for highresolution phylogeographic studies of Colocasia esculenta (L.) Schott (Araceae) and closely related taxa. Mol. Ecol. Resour. 13: 929-937. http://dx.doi.org/10.1111/1755-0998.12128

Chen J, Yu Y and Yang Y (2004). Pollution-free cultivation of Xinmaoyu. Shanghai Vegetables 18: 37-38.

Editorial Board of the Flora of China (1979). Flora Reipublicae Popularis Sinicae, Science Press, Beijing.

Huang X, Ke W, Liu Y, Ye Y, et al. (2012). Chromosomal ploidy identification of taro (Colocasia) germplasm resources. China Veget. 36: 42-46.

Huang X, Ke W, Liu Y, Li F, et al. (2013). Evaluation on elite and rare germplasm resources of taro. J. Changjiang Veget. 27: 85-91.

Huang X, Peng J, Ke W, Liu Y, et al. (2014). Analysis on quality characters of 206 taro (Colocasia esculenta (Linn.) Schott) germplasm resources. J. Plant Genetic Res. 15: 519-525.

Irwin SV, Kaufusi P, Banks K, de la Peña R, et al. (1998). Molecular characterization of taro (Colocasia esculenta) using RAPD markers. Euphytica 99: 183-189. http://dx.doi.org/10.1023/A:1018309417762

Jiang S (2006). Assembly of xerophytic vegetable varieties. Science Press, Jiangsu.

Genetics and Molecular Research 15 (3): gmr.15038108 
Li Q, Yang Y, Li Y and Zhou Q (2004). Studies on genetic diversity and taxology of taro in China. J. Hunan Agr. Univ. (Natural Sci.) 30: 424-428.

Li W, Liu D and Hu X (2011). AFLP finger analysis between cultivars and wild type of taros (Colocasia esculenta) from Yunnan, China. Letters Biot. 22: 855-858.

Lin YS, Kuan CS, Weng IS and Tsai CC (2015). Cultivar identification and genetic relationship of pineapple (Ananas comosus) cultivars using SSR markers. Genet. Mol. Res. 14: 15035-15043. http://dx.doi.org/10.4238/2015. November.24.11

Lu Z, Li W, Yang Y and Hu X (2011). Isolation and characterization of 19 new microsatellite loci in Colocasia esculenta (Araceae). Am. J. Bot. 98: e239-e241. http://dx.doi.org/10.3732/ajb.1100067

Nath VS, Hegde V, Jeeva ML, Misra RS, et al. (2013). Genetic diversity of Phytophthora colocasiae causing taro leaf blight: analysis using start codon targeted (SCoT) polymorphism. J. Root Crops 39: 168-177.

Nunes RSC, Pinhati FR, Golinelli LP, Rebouçaset TNH, et al. (2012). Polymorphic microsatellites of analysis in cultivars of taro. Hortic. Bras. 30: 106-111. http://dx.doi.org/10.1590/S0102-05362012000100018

NusaifaBeevi P, Sreekumari MT and Kumar V (2011). Study of genetic diversity in South Indian taro (Colocasia esculenta (L.) Schott.) using random amplified polymorphic DNA markers. J. Root Crops 37: 162-167.

Quero-Garcia J, Noyer JL, Perrier X, Marchand JL, et al. (2004). A germplasm stratification of taro (Colocasia esculenta) based on agro-morphological descriptors, validation by AFLP markers. Euphytica 137: 387-395. http://dx.doi. org/10.1023/B:EUPH.0000040521.00303.ac

Sardos J, Noyer J, Malapa R, Bouchet S, et al. (2012). Genetic diversity of taro (Colocasia esculenta (L.) Schott) in Vanuatu (Oceania): an appraisal of the distribution of allelic diversity (DAD) with SSR markers. Genet. Resour. Crop Evol. 59: 805-820. http://dx.doi.org/10.1007/s10722-011-9720-7

Tamura K, Stecher G, Peterson D, Filipski A, et al. (2013). MEGA6: Molecular Evolutionary Genetics Analysis version 6.0. Mol. Biol. Evol. 30: 2725-2729. http://dx.doi.org/10.1093/molbev/mst197

Trimanto, Sajidan and Sugiyarto (2010). Characterisation of taro (Colocasia esculenta) based on morphological and isozymic patterns markers. Nusantara Bios. 2: 7-14.

Wołkowicz T, Wolaniuk N, Zacharczuk K, Gierczyński R, et al. (2014). [Development of molecular PCR-RFLP test for identification of the epidemic strain of $Y$. enterocolitica bioserotype 1B/O8 circulating in Poland since 2004]. Med. Dosw. Mikrobiol. 66: 89-98.

Wu Z and Raven PH (2010). Flora of China, Science Press, Beijing.

Yang BG and Kong QD (1998). A study on the classification of the germplasm resources of taro. In: Ethnobotany and genetic diversity of Asian taro: focus on China. Proc Symp Ethnobotanical and Genetic Study of Taro Diversity in China, 26-31. Chinese Academy of Agricultural Sciences, Beijing.

Zhang DX and Zhang GM (1998). Preliminary studies on evolution and classification of taro (Colocasia spp.) in China. In: Ethnobotany and genetic diversity of Asian taro: focus on China. Proc Symp Ethnobotanical and Genetic Study of Taro Diversity in China, 32-45. Chinese Academy of Agricultural Sciences, Beijing, China.

Zhang G and Yang Z (1984). Studies on chromosome number of main cultures of Colocasia esculenta in China. Acta Hort. Sin. 11: 187-190.

Genetics and Molecular Research 15 (3): gmr.15038108 